\title{
Context modeling: Context as a dressing of a focus
}

\author{
Juliette BREZILLON, Patrick BREZILLON \\ LIP6, University Paris 6, 104 avenue du Président Kennedy, 75016, Paris, France \\ \{Juliette.Brezillon, Patrick.Brezillon\}@lip6.fr
}

\begin{abstract}
Contextual Graphs are a context-based formalism used in various real-world applications. They allow a uniform representation of elements of reasoning and of contexts for describing different human tasks such as diagnosis and interpretation. The representation of reasoning by Contextual Graphs supposes an efficient representation of the knowledge associated with a reasoning. Extending the previous view of (1) context relative to a focus, and (2) context as composed of external knowledge and contextual knowledge, in this paper we go a step further by proposing a description of the focus in terms of the instantiation of the contextual elements, which are drawn from domain knowledge. We present the results of this study in a real-world application that we are working on currently, namely the self-evaluation of drivers' behaviors in a situation presented within a large spectrum of contexts.
\end{abstract}

Keywords: Knowledge representation, Contextual knowledge, Proceduralized context, Road safety, Drivers' behavior modeling, self-training

\section{Introduction}

Brézillon and Pomerol [5] defined context as "that which constrains something without intervening in it explicitly." We now consider the "something" by extension as a focus for an actor. Several elements justify this definition, the three main elements being that (1) context is relative to the focus, (2) as the focus evolves, its context evolves too, and (3) context is highly domain-dependent. As a consequence, one cannot speak of context in an abstract way.

Next, we can show that the focus allows the division of context into external knowledge and contextual knowledge [3]. The latter constitutes a kind of tank where the contextual elements are to some extent related to the focus in a flat way, whereas the former has nothing to do with it. At this conceptual level, the focus acts as a discriminating factor on knowledge, like in social networks [4]. The focus evolves because a new event occurs (e.g. an unpredicted event) or as a result of a decision made at the previous stage of the focus. Consequently, the notion of context impacts more on the relationships between knowledge pieces than upon the pieces themselves.

At present, our research on context is organized along two axes, namely reasoning as represented in Contextual Graphs, and knowledge as represented through the instantiation of a part of contextual knowledge. The formalism of contextual graphs has been used in several real-world applications. In this paper, we present an application addressing the question "How can we model context as a dressing of a 
focus?", which allows the identification of differences between the behavior prescribed by procedures and the effective behaviors of actors. This is completely in line with the prescribed and effective tasks identified by Leplat [13]. We observe this situation in our applications such as road safety (an aid for drivers' self-evaluation ), medicine (an aid for users to query reformulation in a grid environment) and software engineering (for form filling and for the assembly of pieces of software).

The second axis tries to go beyond the distinction between external and contextual knowledge. To address the current status of the focus, the actor selects a subset of contextual knowledge that is assembled, organized, and structured within a proceduralized context. In terms of Contextual Graphs, the proceduralized context is an ordered series of instantiated contextual elements. The two keywords here are, first, instantiation, which is also the link between the two axes, and, second, the comparison of the proceduralized context to a buffer between the focus and contextual knowledge.

Applying these observations to real-world applications, context acts like an interface between domain knowledge and the focus. We investigated a problem in road safety, namely the representation of a simple crossroad in terms of a situation dressing. Domain knowledge contains elements like roads, lanes, traffic lights, countryside, city, lights, etc. To define a specific intersection, we must contextualize domain knowledge ("Place" = "City", "Traffic lights" = "No", etc.). Thus, when an element like "Place" is instantiated by "City," some other domain elements become irrelevant (e.g. "Type of field at the corner") and others must be instantiated (e.g. "Type of building at the corner" must be defined by the number of floors, the color, where the door is, etc.). This kind of dressing corresponds to a contextualization of the situation (i.e. the intersection). This contextualization, and thus we go back to the first axis on reasoning, leads to two types of inferencing rules. The first type concerns integrity constraints (e.g. "Period of the day" = "Night" implies that it is not daylight in France). The second type is composed of rules about what one must do in a given context (e.g. "Period of the day" = "Night" implies that "Car lights" must be instantiated to "Switch on"). This constitutes the theoretical behavior that actors must display in the specific context of the situation, that is for the given situation dressing.

In this paper we discuss the relationships between the focus and its context in the framework of our application for road safety. More precisely, we study how contextual elements, relying on domain ontology, could define what is necessary for the focus to be defined.

Hereafter, this paper is organized as follows: in Section 2, we present briefly the ACC project in which this work is ascribed. In section 3, we show that context and situation are two different concepts: the context of a situation appearing like "a set of instantiated contextual elements". In section 4, we explain that viewing context as instantiated contextual elements facilitates the definition of a situation dressing, and in Section 5 we explain the modeling of drivers' behaviors. We conclude by giving some possible extensions for this approach. 


\section{2 "Behavior dressing" and "Situation dressing"}

\subsection{GADGET}

The GADGET project (this is the acronym for "Guarding Automobile Drivers through Guidance Education and Technology") is a European project on road safety [10]. It proposes a representation of drivers' behaviors at four levels. This project originates in the work of van der Molen and Bötticher [11] who split driving tasks and driving behavior into three hierarchical levels: a Strategic level, a Maneuvering or tactical level, and a Control or operational level. In the GADGET matrix, later also referred to as the GDE matrix [14], a fourth level - the Political level - was added above the other three. Our work assumes that the GADGET methodology must be considered with a contextual dimension too. The GADGET variables, which allow the description of car-driving activity, are contextual elements for drivers' behaviors. This arises from the fact that variables like "Traffic regulation" and "Travel planning" are contextual elements that may constrain the focus (e.g. "Drive slowly because you're not in a hurry"). Our objective is to improve the GADGET representation of drivers' behaviors to produce a coherent and complete picture, thanks to the notion of context. Another assumption is that a decision support system would benefit from a driver's experience when it fails to assimilate the driver's behavior to a known behavior. The system could identify a good or bad behavior for the driver. When it fails and cannot identify a driver's behavior, it enters a learning phase for the unknown behavior and for the acquisition of missing knowledge. Once the driver's behavior is identified, the system evaluates if this behavior leads either to a normal or a critical situation. In the latter situation, the system may propose a change of behavior to the driver by explaining how to move from this potentially dangerous behavior to a safer behavior (and thus go back to a normal situation), and then it is the driver who will learn something.

\section{2 "Behavior dressing"as an extension of GADGET}

The first step of our work was to supplement the variables used in the GADGET methodology. In this section, we introduce the variables that we added. According to our viewpoint, the missing variables were:

- Personal variables relating to the driver, such as his/her gender, age, type of employment, whether or not he/she had passed his/her driving license first time, how long he/she has ha a license, how much he/she drives daily, and if he/she is a professional driver, the type of driving work.

- Motivational variables relating to driving, like whether or not the driver is trying to save gasoline, whether or not the driver always drives as quickly as possible, whether or not the driver always tries to avoid damaging the car, whether he/she drives to relax, and whether the driver likes car races .

- Variables dealing with climatic conditions, such as whether or not the driver likes to drive in the rain, in the fog, or when there is snow or ice. 
- Variables describing the driver's physical or emotional state, such as whether or not he/she is tired or stressed, etc.

- Variables describing the driving environment, such as whether or not the driver takes care of children and animals on the road, or if there are animals or children or adults with him/her in the car.

- Variables describing the car, such as its color (insurance companies say that drivers of red cars are aggressive), if the driver takes care of the car, if the driver considers the car as a functional object or a family member, the make of car, the type of vehicle (car, bike, motorbike, bus) and if the car is a company car.

All these variables are not GADGET variables, whereas we think they are important for the description of driving activity. For example, the "gender" variable was not present in descriptions of driving activity; however studies show that women and men do not drive in the same way. All the personal variables that we included are important because they describe the driving experience and the driver's social background, even if they are not directly related to the driving task itself. They were not previously taken into account whereas a person who drives every day for his job, for example, does not drive in the same way as a person who drives only for vacations.

The second step of our work was to model the driver's behavior simultaneously at the four levels of the GADGET methodology. We found in the literature that most of the studies based on the GADGET methodology focused on one level. For example, one study focused on the driver's reaction time at the operational level. Another study focused on the driver's mental representation of the road at the tactical level.

We make the assumption that in order to model the driving task, it is necessary to take into account all four levels of the GADGET matrix. Indeed, in a hierarchical structure, all the upper levels influence the lower levels. For example, if the driver is under time pressure, he/she will not drive as he/she usually does, and maybe he/she will take some risks that he/she wouldn't normally take . If we model this case at the tactical level for example, we just model the fact that the driver is taking serious risks whilst driving, so we will say that he/she is a dangerous driver, and we will not consider that he/she is very stressed.

To take into account all the levels at the same time, we proceed as follows: we consider all the GADGET variables independently of the levels where they are, and regroup them by type of variable. There are two kinds of classification: the variables are repeated (this has a direct influence on the highest level) or the variables describe the same aspect of the driving task.

All the variables are related to some extent to the driver's behavior (the focus), but not all the variables concern a specific driver. Thus, the next step is to see how the contextual elements (i.e. the variables) relating to a specific driver are instantiated, that is what the value of these contextual elements is for that specific driver. The key point here is not that the contextual elements are instantiated but how these instantiations are interrelated. This is what we call the proceduralized context [5]. Thus, this work has two objectives: (1) identification of classes of driver from real data (driving behaviors), and (2) identification of the class corresponding to a given driver. 


\section{3 "Situation dressing"}

We propose to model driving behavior using a dual approach. First, there is a global approach, which uses the presentation of choices made by other drivers with a similar profile (e.g. choice of method, choice between alternatives) for the same task. This type of modeling considers a driver as a "collective being", that is modeled from a set of users or user categories acting in a similar way $[1,7,15]$. Such a driver model can be described using a network of concepts and relationships between these concepts (e.g. Bayesian nets or hidden Markovian models). Second, a local (or specific) approach considers the driver using the role he/she plays, the behavior he/she displays, the driving task, the situation and the contexts in which these occur. Thus, the driver is represented as:

- A "variable being," because the driver may have different goals and behaviors during the accomplishment of the task,

- A "unique being," because the driver has specific knowledge, centers of interest, expressed preferences, etc., and

- The being responsible for a driving task that has been contextualized.

The association with a cognitive (local) approach allows the problem to be placed within a larger space, which mainly concerns the representation of the driving situation the driver faces. In other words, we are trying to model driving behaviors from a dual viewpoint: an external view of the driver (from a driver classification, a behavior typology, etc.) and a direct, internal view of the given driver. Our intuition is that qualified driving behavior must rely on the driver's double objective and subjective approaches, using:

- A typology of drivers (Which type of driver am I? Which type of driver should I be?)

- A typology of situations (normal, critical and pre-critical situations)

- A typology of practices ( How do I drive? How should I drive?).

Thus, a driver accomplishing a driving task is considered as a triple\{driver, situation, practice\}. Such a representation is highly contextualized. Nevertheless, our approach relies on some methodologies already proposed in the literature, especially the GADGET methodology, the scenarios proposed in TRAINER and methodology developed for the training of driving instructors [9]. The driver should be able to tackle the following scenarios:

- Identify the type of behavior (and its associated context) the driver is involved in, to evaluate the degree of danger presented by that behavior, and find a way to regain a normal behavior;

- Identify the situation and the driving context, evaluate how critical the situation is, identify pre-critical situations and find the best way to modify his/her behavior in order to regain a normal driving situation .

- Stay vigilant to the behavior of other drivers, detect an abnormal behavior, evaluate a danger for him/her and consequently adapt his/her behavior. (Note that not all drivers perceive a danger identically.)

- The time constant for the driver's response is an essential parameter in our proposal. For example, being vigilant to other drivers' behaviors is a routine task in driving (with a long time constant), whilst adapting his/her behavior to the danger presented by other drivers may have a very short time constant (often less than one second). 
A scenario corresponds to the evolution of a situation (and its context) assimilated to a series of situations. A scenario is a path in the situation space with pre-critical situations playing a role similar to a bifurcation point [17]. A pre-critical situation has two potential successors, a normal situation if the driver correctly modifies the driving plan or a critical situation if the driver does not react correctly.

Driving behaviors are represented in the context-based formalism called Contextual graphs that provide a uniform representation of elements of reasoning and of contexts [3]. The principles of contextual-graph building for driving are the following. A contextual graph represents the different behaviors that a driver may present in a given driving situation (a crossroads in the following case study). A path in this graph represents the driver's behavior in that driving situation for a given context (i.e. instantiated elements like "Status" = "In a hurry" and "Weather" = "Rain"). Contextual elements are instantiated because their value is explicitly considered in the decision making process. A driver can exhibit different behaviors for a given scenario, and the same behavior may appear in different scenarios. Thus, drivers' behaviors are represented in a dual space (situation space and a behavior space), and scenarios are the bridge between these two spaces. An intelligent tutoring system using such a database can (1) identify the driver class to which the user belongs, (2) select a situation in which the user has some weaknesses, (3) establish the scenario for which the user may learn how to modify his behavior, (4) follow the driving task realized by the user and anticipate pre-critical situations that may be encountered by the user, and (5) propose ways of correcting the user's behavior.

Domain knowledge, which defines driving situations, is represented by contextual elements. A single context for the situation is an instantiation of a (large) part of these contextual elements. In the next section we discuss the modeling of the situation and of drivers' behaviors for the crossroads case study.

\section{Case study}

\subsection{Situation description}

Consider the real traffic situation--a simple crossroads - for which we are trying to analyze all the driving situations that could occur. We assume that only two cars arrive at the crossroads. The Highway Code gives the "driver model": "Give right of way to the car coming from your right." Because the system must support a given driver, we choose to select the viewpoint of the driver of the black car (coming from the bottom in Figure 1). We will analyze all possible scenarios, first, according to where the white car is coming from (from the left, the right or in front of the black car), and second, according to the movements of the two cars (turn left, go straight ahead, or turn right) at the crossroads (see Figure 1).

Domain knowledge corresponding to this situation is described by contextual elements that are organized in a hierarchy with instantiations of some of them. A piece of this hierarchy is given as follows:

\begin{tabular}{|ll} 
Physical elements & (e.g. Environment) \\
Technical elements & (e.g. Type of crossroad structure)
\end{tabular} 
\begin{tabular}{|ll} 
Moment elements & (e.g. Day) \\
Driving elements & (e.g. Vehicle) \\
Human elements & (e.g. Physical aspects)
\end{tabular}

B

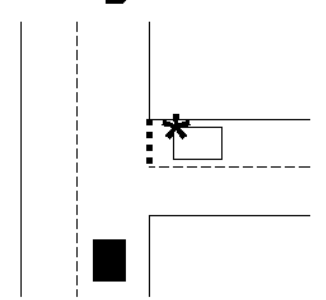

A

Figure 1: The chosen driving situation

Such contextual elements allow us to describe the situation at the desired level of detail. According to the hierarchy of domain knowledge, there are various levels of detail in the representation of a situation. For example:

Physical element $>$ Environment $>$ City $>$ Village

The granularity depends on the driver's focus and provides a way to know the driver's level of situation awareness. A driver may not pay attention to the fact that "City" = "Village" and thus may miss some important points he/she needs to take into account in terms of his behavior (e.g. "Reduce speed when driving through a village").

The instantiation of the selected contextual elements (e.g. "Traffic light" = "No") leads us to specify a context for the situation. Changing the instantiation of "Traffic light" = "No" to "Yes" leads us to another context, and the situation, i.e. the crossroads, must be perceived differently by the driver, who must adapt his/her behavior. The instantiation of the contextual elements is a contextualization of the situation. The instantiation set of the contextual elements is called situation dressing.

\subsection{Dressing of the situation}

A contextual element has an instance, which can be either a value or another contextual element. This enables us to make explicit the level of detail of the description but supposes a mechanism for acquiring new concepts incrementally because "we don't know what we need before we have to use it" [11]. This leads to a mechanism of incremental acquisition. (We are currently implementing such a piece of software.)

Another consequence is that a contextual element is itself a list of elements, and a recursive one. The situation above is described (very partially) as follows:

Physical elements

Environment $=$

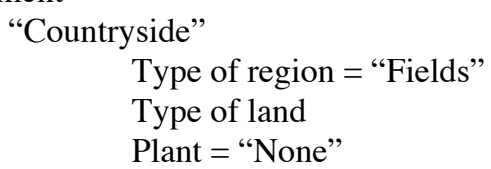




$$
\text { "City" Pasture = "No animal" }
$$

The dressing of a situation represents a context for the "crossroads" situation. Changing one instantiation, e.g. "Field at the right corner of the intersection crossroads" = "Corn field" to the instantiation "Reaped field", leads to another context, and the driver's behavior must be modified (a reaped field lets the user see if the white car coming from the right is approaching the intersection or stopping at the intersection, whereas a corn field means that what is on the right side of the road is not visible.

The situation dressing allows to present the same situation in various contexts (changes of instantiation of any contextual element, or instantiations chosen randomly). Thus, the user must always be careful in analyzing the situation and learn to manage the situation with its context. This is a way of developing situation awareness.

If domain knowledge imposes a structure on contextual elements as discussed above, contextual elements must also obey another organization that depends on the relationships existing between them. We summarize this in Figure 2.

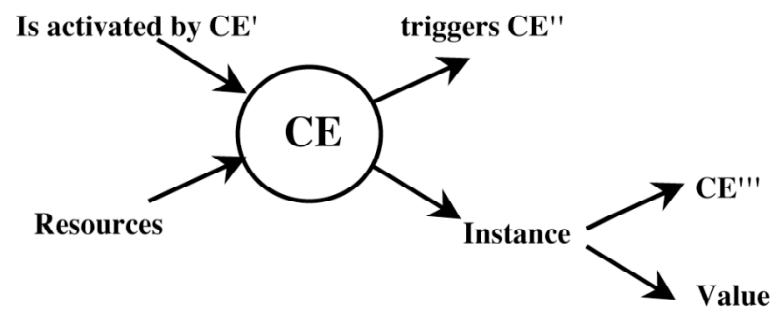

Figure 2: Relationships between Contextual Elements (CEs)

We already have seen that an instance may be a value or another contextual element (CE). The determination of a value is made from different resources: a sensor (e.g. the temperature), a database (e.g. a user's profile for the user's gender), a computation (e.g. the value of a dollar in euros), or questions to the user (e.g. "Do you like going fast?"). The last point is that the instantiation of a contextual element may lead to another contextual element being triggered or inhibited . For example, choosing "Countryside" implies that "Type of building at the right hand corner" is not relevant, but that it is mandatory to instantiate "Type of field." In the same spirit, the instantiation "Corn" for the contextual element "Type of field" means that (1) corn is tall and hides the view of the white car, (2) visibility is strongly reduced, and (3) the driver must drive carefully. This implies that two types of rules must be considered. In [19], we present a piece of work on the design of a context manager for managing contextual elements. 


\subsection{Situation dressing refinement}

\subsubsection{Integrity constraints}

Integrity constraints are rules that describe relationships between contextual elements and their instantiation. For example, we can extend the previous example of the "Environment" with rules like:

IF "Item at the right corner" = "Field"

THEN Look for "Season"

IF "Season" = "Summer",

THEN look for "Type of field"

ELSE "Type of field" = "Reaped"

IF "Type of field" = "Corn"

THEN "Visibility of the road on the right" = "Nil"

ELSE "Visibility of the road on the right" = "Good"

(The last line would be refined by taking into account, say, "Weather" (for fog).

Another group of integrity rules concerns the relationships between contextual elements that rely on common sense knowledge. For example:

IF "Moment of the day" = "Night"

THEN "Weather" cannot be "Sunny"

IF "Weather" = "Rainy"

THEN "Road state" must be "Wet"

Making explicit such rules in a decision support system allows the consideration of the situation in a coherent context in order to identify the important contextual elements that are able to explain a situation.

\subsubsection{Inference rules about the driver's behavior}

Rules about the driver's behavior do not concern directly the instantiation of contextual elements but the relationship between instantiation of contextual elements and the driver's behavior. Examples of such inference rules are:

IF "Road state" = "Wet" or "Weather" = "Fog" or "Visibility" = "Weak"

THEN "Car speed" = "Reduced" and "Driver status" = "Vigilant"

IF "Field on the right" = "Reaped"

THEN "Look to see if a car is coming from the right"

IF "Sidewalk (Pavement en GB)" = "Small" and "Crowded"

THEN "Watch to see if a pedestrian in a hurry goes on the pavement into the road"

IF "Type of day" = "Working day"

THEN IF "Time" = "Morning" and "Near crossroads" = "School"

THEN "Pay attention to children going to school" "Anticipate needing to brake quickly"

IF "White car" = " Emergency vehicle"

THEN " Give it right of way" 
The model of the theoretical behavior of drivers arriving at a simple intersection in the Highway Code corresponds to the unique rule:

IF "Car on the road to the right" THEN "stop and give it right of way".

This is the rule in the Highway Code that corresponds to the situation only. We have shown here that the contextualization of the situation (it is night, it is raining, etc.) leads to a richer model, not of the drivers but of their behaviors. Context allows consideration of a task within its environment (a contextualized task). Another observation is that if the model of the theoretical behavior is unique for a situation, the model of the "prescribed behavior" of drivers is context-dependent and thus there is a specific model of prescribed behavior for each context. This means that the "distance" between the prescribed task and the actual task can be defined more precisely. With respect to the companion paper [2], we are able to propose a task model (i.e. the prescribed task) that describes a contextualized task. Now we will present the model of drivers' effective behaviors, that provides the second space for describing our problem, alongside the situation space already discussed in this section.

\section{Model of drivers' behaviors}

We represent the evolution of a situation for a given driver as a movement through a series of situations represented in a discrete space of situations. Figure 3 represents five scenarios that can be derived from the first traffic situation given in Figure 1.

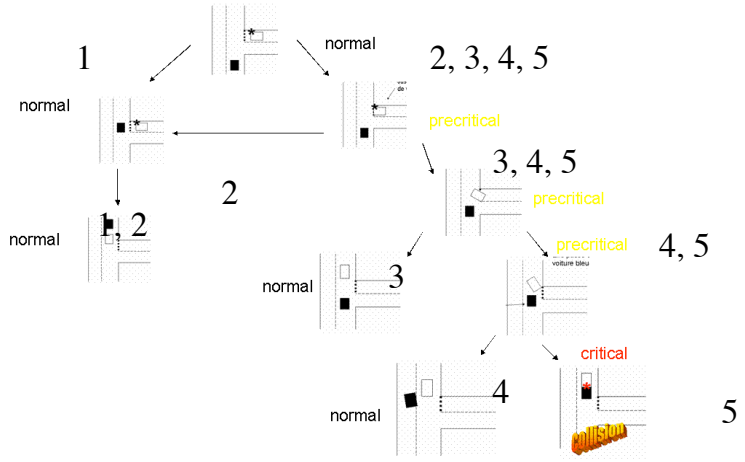

Figure 3: Graph of all the situations that can be derived from the first one

Scenario 1 corresponds to the normal situation. The black car goes forward and the white car waits until the black car has passed and then turns right after it.

In scenario 2, the white car goes forward a little just to reach the road marking. There are several hypotheses for why the driver of the white car goes forward a little. For example, the driver of the white car might think he/she has time to complete his/her action (turn right before the black car) but abandons the idea rapidly. Another reason could be that the driver wants to look behind the black car to see if any other vehicle is coming. The driver of the black car reduces speed, observes the 
white car, and, if the white car is no longer moving, decides to maintain his/her driving plan but is careful. Afterwards, the white car turns right, and the second scenario corresponds to the first scenario (see Figure 3).

In scenario 3, the white car goes forwards until the junction markings and the driver decides to act before the black car arrives. Conversely, the driver of the black car had a different interpretation of the situation and anticipated that the other driver would stop at the road marking. However, the driver of the black car is prudent in this situation and is aware of the risk of a critical situation and quickly understands the the driver of the white car's intention when the white car goes forward. Thus, the driver of the black car has time to brake. As we assume that there is no other vehicle in the situation, the driver of the black car can brake easily without posing a risk to the other cars behind him/her. The black car brakes and stops (or at least reduces sufficiently its speed), lets the driver of the white car finish turning, allows some safe distance between the two cars and goes forward, after the white car.

The fourth scenario supposes that, on the one hand, the white car goes forward to pass in front of the black car as in scenario 3, and, on the other hand, the driver of the black car interprets the modified situation differently, thinking that the driver of the white car will wait before moving because he/she does not have right of way. Realizing that the driver of the white car is not behaving as expected, the driver of the black car is surprised and has only a short time to react. The driver of the black car tries to brake, but not quickly enough . To avoid a collision between the two cars, and because there are no other vehicles in the area, the driver of the black decides to overtake the white car and to change lane.

The fifth and last scenario is a variant of the fourth scenario. The driver of the black car tries to brake, but not quickly enough and has no time to change lanes (or can not do it) and a collision between the two cars thus happens.

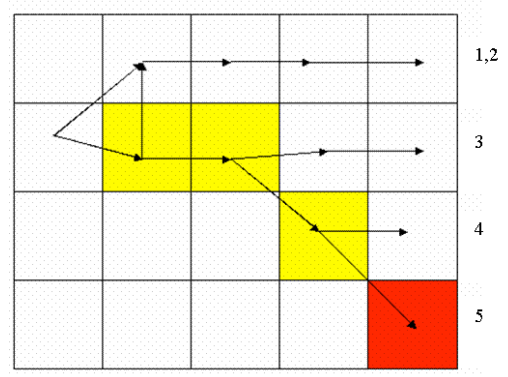

Figure 4: The five scenarios at their different steps (white squares represent normal situations, pre-critical situations are in grey and the critical situation is in the dark square) in the situation space

Figure 4 gives a representation in the situation space of the different situations and scenarios identified in Figure 3. There is one critical situation and three precritical situations (in grey and corresponding to scenarios 2, 3, 4), which leads to five scenarios.

Figure 5 represents drivers'behaviors in the scenarios shown in Figure 4 in the contextual-graphs formalism ( The key/caption for Figure 5 is given in Table 1.) Note that the contextual graph contains only the black-car driver's behaviors. The 
description is more extended (e.g. there are two behaviors that lead to scenario "5") because we have chosen the black-car driver's viewpoint and not the viewpoint of an external observer. This will be the topic of another paper.

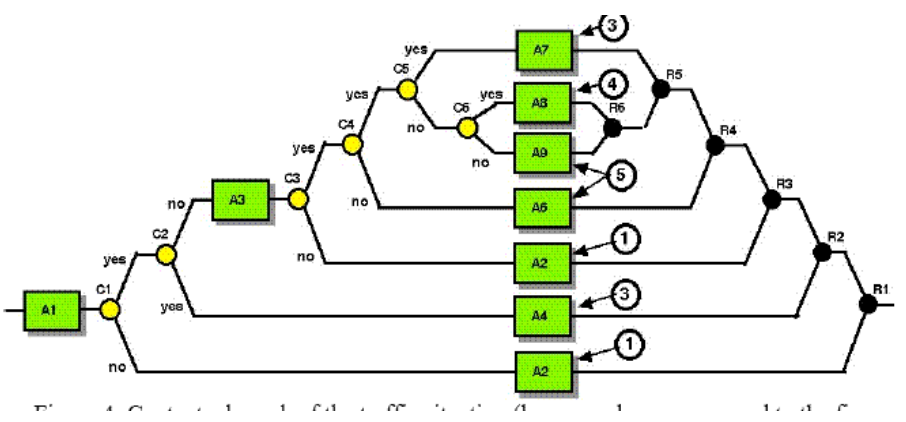

Figure 5: Contextual graph of the traffic situation (numbers in circles correspond to the five scenarios in Figures 3 and 4). Elements are defined in Table 1.

\begin{tabular}{|c|c|}
\hline Contextual element & Is the white car stopping? \\
\hline C1 & Is the white car going forward? \\
\hline C2 & Can I let the white car go forward? \\
\hline C3 4 & Can I overtake the white car on the left? \\
\hline Action & Definition \\
\hline A1 & Maintain the same behavior \\
\hline A2 & Interpret the behavior of the driver of the white car \\
\hline A3 & mote that the driver of the white car is stopping at the road \\
\hline A4 & Brake enough to let it go forward \\
\hline A5 & Change lane and overtake \\
\hline A6 & Evaluate the situation \\
\hline A7 & Try to brake strongly \\
\hline
\end{tabular}

Table 1: Contextual elements and actions while negotiating the crossroads in Figure 5

\section{Conclusion}

This approach is interesting in that a given situation (the crossroads in our study) can be proposed in a large number of contexts. Thus, although the situation is unique, the large spectrum of contexts will force a driver to be attentive all the time and aware of the specificity of the situation each time. We believe that our approach develops a sense of situation awareness within training.

Making context explicit within our application enables user-centered management of domain knowledge. The context is domain knowledge, and the focus guides the distinction between external knowledge and contextual knowledge. The former has nothing to do with the focus (e.g. what is a motorway? in our study), when the former is to some extent related to the focus (e.g. traffic light and lane number). A subset of contextual knowledge is extracted, assembled and constitutes the proceduralized 
context. This is concretely obtained by combining and inferring relationships between the selected contextual elements. For example, a simple crossroads supposes a special sign before the intersection, but no traffic light. What is important here is that the main distinction between contextual knowledge and the proceduralized context is the instantiation of the contextual elements in the proceduralized context. Instantiation leads to the application of a set of rules (integrity constraints and inference rules), the latter providing a task model, not of the domain, but of how to behave in the contextualized situation. In this sense, our approach provides a way to generate automatically a contextualized model of the driver's behavior for the actual situation, not of the abstract situation.

Endsley [8] established the well-known definition of Situation Awareness with its three levels: (a) perception of elements, (b) comprehending what those elements mean and (c) using that understanding to project future states. Our approach is ascribed in this realm. The notion of focus defining the related contextual elements that are relevant to the current context is a factor that can improve perception in Endsley's first level. Making the distinction between the selected contextual elements and their instantiations explicit may be associated with the second level (i.e. the understanding of the meaning of the elements.) The identification of the rules of "good behavior" (and by contrast of "bad behavior") in the context of the situation allows decision making to be efficient and the third level to be predicted.

This paper gives a new vision of the classical dichotomy "prescribed task versus effective task." We have shown that rules, which are deduced from the instantiation of the contextual elements, lead to a task model that relates to the contextualized situation, not the situation alone. This is important in terms of drivers' behaviors because if the Highway Code addresses the situation (at a simple intersection), the vehicle coming from the right has right of way), the task model that arises from inference rules is able to adapt to the contextualized situation. For example, if the driver coming from your right stops his vehicle and indicates that you can go on, it is not necessary to stop and wait. Thus, the driver will learn a set of operational rules instead of a general rule. In other words, our approach is a means for drivers to develop an efficient model of practice instead of a task model (i.e. a theoretical model). It is more important to learn how to use a rule rather than just to learn the rule.

Acknowledgments. The ACC project is supported by PREDIT GO3 and the French Ministry of Transport (Grants SU-05-276), mainly in terms of the funding of a Ph.D.Thesis. We also want to thank the members of the ACC project, especially T. Artières, P. Gallinari and Ch. Tijus. Information on the ACC project can be found at www-poleia.lip6.fr/ jbrezillon.

\section{References}

[1] Blanchard et al. (2005) Blanchard, J., Petitjean, B., Artières, T. and Gallinari, P. (2005) Un système d'aide à la navigation dans des hypermédias In EGC 2005, Paris. 
[2] Brézillon, P. (2007) Context modeling: Task model and model of practices. Proceedings of the $5^{\text {th }}$ International and Interdisciplinary Conference on Modeling and Using Context, CONTEXT-07 (This volume).

[3] Brézillon, P. (2005) Modeling users' practices in contextual graphs. In: A. David (Ed.) Organisation des Connaissances dans les Systèmes d'Informations Orientés Utilisation. Contexte de Veille et d'Intelligence Economique (ISKO-2005). Presse Universitaire de Nancy, pp. 77-96

[4] Brézillon, P. (2005b) Role of context in social networks. Proceeding of the 18th International FLAIRS Conference, Invited Special Track "AI for Social Networks, Social Networks in AI", AAAI CD Rom, pp. 20-25.

[5] Brézillon P. and Pomerol J-Ch. (1999), Contextual knowledge sharing and cooperation in intelligent assistant systems. Le Travail Humain, 62(3): 223-246.

[6] Brézillon, P., and Tijus, C. (2006) Increasing The rationality of cognitive tasks with contextual graphs . Proceedings of the 8th European Meeting on Cybernetics and Systems Research, Vienna, Austria, April 18-21.

[7] Brusilovsky, P. (2001) Adaptive hypermedia, User Modeling and User Adapted Interaction, Ten Year Anniversary Issue (Alfred Kobsa, ed.) 11 (1/2), 87-110

[8] Endsley, M.R. (1995), Toward a Theory of Situation Awareness in Complex Systems. Human Factors.

[9] Gregersen, N.P. and Bartl, G. (2005) Young car drivers: Why are they over represented in car accidents? How can driver training improve their situation. VTI report 409A, Linkoping: Swedish National Road and Transport Institute.

[10] Hatakka, M., Keskinen, E., Gregersen, N.P., Glad, A. \& Hernetkoski, K. (2002) From control of the vehicle to personal self-control; broadening the perspectives to driver education. Transportation Research, Part F, 201-215.

[11] Henninger, S. (1992) The knowledge acquisition trap. Proceedings of the IEEE Workshop on Applying Artificial Intelligence to Software Problems: Assessing Promises and Pitfalls (CAIA-92), Monterey, CA, March, pp. 51-57.

[12] Laird, J. E. and Newell, A. (1987). Knowledge level learning in SOAR, Proceedings of the Sixth National Conference on Artificial Intelligence, pp. 499-504.

[13] Leplat, J. (1997) Regards sur l'activité en situation de travail - Contribution à la psychologie ergonomique. Paris : Presses Universitaires de France.

[14] Peräaho, M., Keskinen, E. and Hatakka, M. (2003) Driver Competence in a Hierarchical Perspective; Implication for Driver Education. University of Turku, Traffic Research, Turku 2003.

[15] Pohl, W. and Nick, A. (2000) Machine Learning and Knowledge Representation in the Labour Approach to User Modeling, Proceedings of the 7th International Conference on User Modeling, Banff, Canada pp. 197-188.

[16] Siegrist, S. (1999) Rapport GADGET, Formation et évaluation du conducteur, obtention du permis de conduire. Vers une gestion théoriquement fondée du risque routier des jeunes conducteurs. Résultats du projet européen GADGET - Groupe de travail Nº3, Stefan SIEGRIST (ed.) Berne 1999.

[17] Thom, R. (1972). Stabilité structurelle et morphogénèse. Essai d'une théorie générale des modèles. Paris: Inter Editions.

[18] Van der Molen, H.H. and Bötticher, A.M.T. (1988) A hierarchical risk model for traffic participants. Ergonomics.

[19] Vieira, V. Tedesco, P., Salgado, A.C. and Brézillon, P. (2007) Investigating the specifics of contextual elements management: The CEManTIKA approach. Proceedings of the $5^{\text {th }}$ International and Interdisciplinary Conference on Modeling and Using Context, CONTEXT07 (This volume). 